\section{Referências}

1. Budin PC. Le nourrison: alimentation et hygiene. Enfants debile et enfants nes a terme. Paris: Doin; 1900.( http:// www.neonatology.org/classics/default.html )

2. Lubchenco LO, Hansman C, Dressler M, Boyd E. Intrauterine growth as estimated from liveborn birth-weight data at 24 to 42 weeks of gestation. Pediatrics. 1963;32:793-800.

3. Anderson DM. Nutritional assessment and therapeutic interventions for the preterm infant. Clin Perinatol. 2002;29:313-26.

4. Ehrenkranz RA, Younes N, Lemons JA, Fanaroff AA, Donovan EF, Wright $L L$, et al. Longitudinal growth of hospitalized very low birth weight infants. Pediatrics. 1999;104:280-9.
5. Dusick AM, Poindexter BB, Ehrenkranz RA, Lemons JA. Growth failure in the preterm infant: can we catch up? Semin Perinatol. 2003;27:302-10.

6. Anchieta LM, Xavier CC, Colosimo EA. Crescimento de recémnascidos pré-termo nas primeiras doze semanas de vida. J Pediatr (Rio J). 2004;80:267-76.

7. Ziegler EE, Thureen PJ, Carlson SJ. Aggressive nutrition of the very low birthweight infant. Clin Perinatol. 2002;29:225-44.

8. Singhal A, Lucas A. Early origins of cardiovascular disease: is there a unifying hypothesis? Lancet. 2004;363:1642-5.

\title{
Redes multicêntricas e a qualidade da atenção neonatal
}

\author{
Multicentric networks and quality of neonatal attention
}

\author{
Fernando C. Barros' ${ }^{1}$ José Luis Diaz-Rossello²
}

ma das preocupações da saúde pública atual é o aumento dos nascimentos pré-termo no mundo e sua crescente importância como causa de mortes infantis. Nos estudos que demonstram um incremento da prevalência nos Estados Unidos e Canadá1,2, as causas mais invocadas para este fenômeno são o aumento de intervenções obstétricas, o aumento na freqüência de nascimentos múltiplos e as melhorias na qualidade da observação sobre idade gestacional, devido à substituição de cálculos por data da última menstruação pela utilização crescente de estimativas por ultra-som realizado precocemente na gravidez.

No Brasil, também parece estar ocorrendo um aumento de nascimentos pré-termo, como demonstram vários estudos, entre eles os de Ribeirão Preto, SP, e Pelotas, RS. Em Ribeirão Preto ${ }^{3}$, houve um expressivo aumento na prevalência de nascimentos pré-termo, de 7,6\% em 1978-9 para $13,6 \%$ em 1994 . Os autores sugerem que o aumento das cesarianas pode ter sido uma das razões deste aumento, embora seja muito difícil eliminar aqui o problema da causalidade reversa.

Em Pelotas, RS, a prevalência de nascimentos prétermo aumentou entre 1982 e 1993 - de $5,6 \%$ para $7,5 \%{ }^{4}$.

1. Investigador e Docente do Centro Latino-Americano de Perinatologia, OPAS/OMS, Montevidéu, Uruguai. Professor titular de Medicina Social, Universidade Federal de Pelotas (UFPel).

2. Professor, Departamento de Neonatologia, Universidade de la República Oriental del Uruguay. Investigador e Docente do Centro Latino-Americano de Perinatologia, OPAS/OMS, Montevidéu, Uruguai.
Estamos, atualmente, realizando um novo estudo perinatal da cidade, e dados para os primeiros 4 meses do ano de 2004 indicam um aumento muito importante de nascimentos pré-termo, que agora são cerca de $18 \%$. Este incremento parece localizar-se entre recém-nascidos pré-termo grandes - com 35 e 36 semanas de gestação - e ocorre tanto para partos vaginais como para cesarianas. Este achado sugere que devemos considerar como possíveis causas desta nova epidemia de pré-termos todas as formas de interrupção da gravidez, incluindo não somente as cesarianas, mas também induções do trabalho de parto (Barros et al.; dados preliminares não publicados).

Como os recém-nascidos pré-termo são, em qualquer população, responsáveis por uma parcela muito importante da morbidade e mortalidade neonatal e infantil, o assunto abordado pela Rede Brasileira de Pesquisas Neonatais (RBPN) neste número do Jornal de Pediatria - a utilização de corticóides antenatais em trabalho de parto pré-termo é de grande atualidade ${ }^{5}$. O uso de corticóides é reconhecido como uma ação preventiva de alta efetividade e muito baixa utilização. Meta-análises recentes demonstram que o uso de corticóides em trabalho de parto pré-termo, ou previamente à interrupção eletiva pré-termo da gestação, pode reduzir de maneira importante a mortalidade neonatal e morbidades graves, como a doença da membrana hialina e a hemorragia intraventricular. Portanto, é muito preocupante que a recente e aclamada série sobre sobrevivência infantil publicada pela revista Lancet tenha estimado que a cobertura mundial desta intervenção, em casos indicados, seja de somente $5 \%{ }^{6}$. 
Na América Latina, a prevalência da utilização dos corticóides antenatais em trabalho de parto pré-termo não é bem conhecida, pois há poucos trabalhos de base populacional que permitam sua mensuração. Um estudo realizado em Montevidéu, Uruguai, e publicado no Jornal de Pediatria mostrou que, nos hospitais daquela cidade, no ano de 1999, a utilização de corticóides em nascimentos de muito baixo peso foi de $59,7 \%$, sendo de $65,6 \%$ nas instituições públicas e $53,5 \%$ nas maternidades privadas ${ }^{7}$.

Para o Brasil, um trabalho de bom padrão metodológico, com dados do início dos anos 90, realizado em maternidades públicas do Rio de Janeiro, mostrou utilização de somente $4,3 \%$ em trabalho de parto pré-termo entre as idades gestacionais de 28 e 34 semanas incompletas ${ }^{8}$. Mais recentemente, a RBPN publicou informações sobre a cobertura de corticóides antenatais para recém-nascidos de muito baixo peso, em suas maternidades, entre 1998-99, e revelou uma prevalência média de $29 \%$, variando entre 10 e $39 \%{ }^{9}$.

Os autores da RBPN descrevem, neste número do Jornal de Pediatria, as características e a evolução de um grupo de recém-nascidos pré-termo e suas mães, pertencentes a dois grupos - as que receberam e as que não receberam corticóides antenatais ${ }^{5}$. A RBPN é constituída por oito unidades de atenção neonatal localizadas em maternidades universitárias do Sudeste e Sul do país, e sua investigação é um estudo multicêntrico do tipo observacional.

A primeira notícia positiva que nos traz o artigo é que, nesta rede, a utilização de corticóides antenatais em trabaIho de parto pré-termo com menos de 34 semanas foi de $61 \%$, bem maior do que qualquer outro estudo latinoamericano de nosso conhecimento. Os autores descrevem em detalhe as características maternas e dos recémnascidos, incluindo sua morbimortalidade. Existe aqui uma dupla intenção: identificar possíveis fatores de risco maternos para a não-utilização de corticosteróides e avaliar o possível impacto dos corticóides sobre a saúde dos recémnascidos pré-termo. Com relação a este último aspecto, chama a atenção neste trabalho que, no grupo que recebeu corticóides antenatais, não houve redução de membrana hialina e houve aumento de enterocolite necrosante, o que não está de acordo com outros dados da literatura. É possível que os critérios de inclusão utilizados possam ter colaborado para esta situação. É chamativo, por exemplo, que as prevalências de infecção materna e/ou bolsa rota prolongada, diabetes e hipertensão arterial foram bem mais elevadas no grupo que recebeu corticóides.

A comparação de recém-nascidos pré-termo que receberam corticóides com os que não receberam pode dar margem a resultados difíceis de entender, justamente porque se trata de dois grupos diferentes não somente pela utilização ou não de corticóides. As mães que não os receberam podem ter sido aquelas que chegaram em franco trabalho de parto e tarde demais para condutas preventivas (uso de tocolíticos em menos de $2 \%$ ) ou que não preenchiam critérios de eleição para o tratamento.

Os autores utilizaram a análise multivariada, através de regressão logística, para identificar, entre muitas variáveis, aquelas que eram associadas com desfechos como ventilação mecânica, hemocultura positiva e sobrevida. O interesse era verificar se o efeito protetor do uso de corticóides contra morte e uso de ventilação mecânica, e seu efeito facilitador de infecções, mantinha-se mesmo depois de se ajustar para diferenças entre as mães e as crianças que usaram ou não os corticóides, das quais as mais notáveis são peso ao nascer, idade gestacional e as condições do recém-nascido na sala de parto. Neste estudo, a análise mostrou que sim (o efeito se mantém), o que está de acordo com o que conhecemos da literatura.

De qualquer maneira, é aconselhável considerarmos esses resultados com o devido cuidado, tendo em vista os problemas metodológicos inerentes a esse processo. Entre as dificuldades do modelo empregado, pode-se mencionar que algumas variáveis incluídas no modelo eram, claramente, variáveis intermediárias dos desfechos estudados. Nesta categoria estão o uso de oxigênio com 36 semanas, SNAPPE II e ventilação mecânica (para os outros dois desfechos). Além disso, outras variáveis agregadas ao modelo têm relações muito particulares com a exposição estudada - uso ou não de corticóides - e que não foram exploradas com a necessária profundidade nesta publicação. Aqui se englobam: uso de tocolíticos, hipertensão materna e tipo de parto. Além disso, quando realizamos análises multivariadas, sempre existe a possibilidade de não termos incluído no modelo variáveis importantes que poderiam ter modificado a associação entre a exposição e o desfecho. Este confundimento residual é um problema dos estudos de observação e pode ser minimizado por observações repetidas e medições cuidadosas. Portanto, parecenos que os resultados das análises multivariadas devam ser vistos à luz destes senões, como alguns achados interessantes a serem explorados no futuro, com metodologia mais adequada.

Os estudos multicêntricos estão em seu apogeu e podem ser encontrados nas melhores revistas médicas do mundo. São, freqüentemente, ensaios clínicos aleatorizados (RCTs), que aproveitam a vantagem de poder recrutar um grande número de pacientes em pouco tempo naquelas investigações que necessitam grandes amostras. Além disso, o fato de que são estudos realizados em muitos lugares pode aumentar a validade externa do estudo, permitindo uma maior generalização dos resultados.

Mas as redes multicêntricas apresentam uma outra grande vantagem, que é a utilização de registros padronizados de variáveis, que permitem a realização de estudos de observação que avaliam a efetividade de técnicas preventivas ou terapêuticas já testadas em RCTs e apresentam resultados para as populações locais. É deste tipo o presente trabalho, e são muitos os exemplos de redes neonatais internacionais, como as norte-americanas Vermont-Oxford e a Rede Neonatal do Instituto de Saúde Infantil do Instituto Nacional de Saúde (NICHD). Esperamos que a RBPN possa ser ampliada, incorporar novos centros de outras regiões do país e colaborar ativamente na promoção das práticas mais adequadas e nas melhorias da qualidade da atenção neonatal no Brasil. 


\section{Referências}

1. Joseph KS, Kramer MS, Marcoux S, Ohlsson A, Wen SW, Alexander $A$, et al. Determinants of preterm birth rates in Canada from 1981 through 1983 and from 1992 through 1994. N Eng J Med. 1998;339:1434-9.

2. Demissie K, Rhoads GG, Ananth CV, Alexander GR, Kramer MS, Kogan MD, et al. Trends in preterm birth and neonatal mortality among blacks and whites in the United States from 1989 to 1997. Am J Epidemiol. 2001;154:307-15.

3. Bettiol H, Rona RJ, Chinn S, Goldani M, Barbieri MA. Factors associated with preterm births in Southeast Brazil: a comparison of two birth cohorts born 15 years apart. Paediat Perinat Epidemiol. 2000;14:30-8.

4. Horta BL, Barros FC, Halpern R, Victora CG. Baixo peso ao nascer em duas coortes de base populacional no sul do Brasil. Cad Saúde Pública. 1996;12 Supl 1:27-31.

5. Rede Brasileira de Pesquisas Neonatais. Uso antenatal de corticosteróide e evolução clínica de recém-nascidos pré-termos. J Pediatr (Rio J). 2004;80:277-84.
6. Jones G, Steketee RW, Black RE, Bhutta ZA, Morris SS. How many child deaths con we prevent this year? Lancet. 2003;362: 65-71.

7. Matijasevich A, Barros FC, Forteza C, Diaz-Rossello JL. Atenção à saúde de crianças de muito baixo peso ao nascer de Montevidéu, Uruguai: comparação entre os setores públicos e privado. J Pediatr (Rio J). 2001;77:313-20.

8. Krauss Silva L, Pinheiro da Costa T, Reis AF, Iamada NO, Azevedo AP, Albuquerque CP. Avaliação da qualidade da assistência hospitalar obstétrica: uso de corticóides no trabalho de parto prematuro. Cad Saúde Publica. 1999;15:817-29.

9. Leone CR, Sadeck LSR, Vaz FC, Almeida MFB, Draque CM, Guinsburg R, et al. Brazilian Neonatal Research Network (BNRN): Very Low Birth Weight (VLBW) infant morbidity and mortality. Pediatr Res. 2001;49:405A.

\title{
Quando devemos iniciar a fototerapia em recém-nascidos pré-termo?
}

\author{
When should we start phototherapy in preterm newborn infants?
}

\author{
Maria Fernanda B. de Almeida*
}

\begin{abstract}
A hiperbilirrubinemia indireta é encontrada em praticamente todos os recém-nascidos pré-termo, principalmente nos de muito baixo peso. Estudos evidenciam que a grande quantidade de glóbulos vermelhos com sobrevida diminuída, o aumento da circulação êntero-hepática da bilirrubina e a deficiência na conjugação hepática da mesma são as condições fisiológicas mais importantes que explicam a hiperbilirrubinemia. Adicionalmente, a demora na introdução da dieta enteral, comum nos prematuros criticamente doentes, pode limitar o fluxo sangüíneo intestinal e intensificar a reabsorção êntero-hepática da bilirrubina. Assim, devido à imaturidade eritrocitária, hepática e gastrintestinal, a icterícia "fisiológica" é mais intensa do que a do termo, com concentração de bilirrubina total entre $10 \mathrm{e}$ $12 \mathrm{mg} / \mathrm{dl}$ no quinto dia de vida, podendo não atingir valores normais até o final do primeiro mês ${ }^{1}$.

Uma das causas específicas mais freqüentes inclui os extravasamentos sangüíneos, seja por hematomas extensos em membros superiores e inferiores devido a parto traumático, seja por hemorragia intraperiventricular, especialmente naqueles com idade gestacional inferior a 34
\end{abstract}

* Professora adjunta, Disciplina de Pediatria Neonatal, Universidade Federal de São Paulo (UNIFESP), São Paulo, SP. semanas. Entretanto, apesar da investigação apropriada da hiperbilirrubinemia indireta segundo a história e a evolução no recém-nascido pré-termo, a grande maioria não apresenta uma etiologia determinada. Além disso, os pacientes submetidos a cuidados intensivos podem apresentar a associação de fatores facilitadores da impregnação bilirrubínica em nível cerebral, tais como hipoxemia, acidose, hipotermia, hipoalbuminemia, hipercapnia, entre outros ${ }^{2}$.

Apesar do encontro quase universal da hiperbilirrubinemia indireta no recém-nascido pré-termo, na década de 90 foram publicadas várias pesquisas que não demonstram uma relação causal entre hiperbilirrubinemia dentro de limites controlados (ou seja, bilirrubina total em média inferior a $15 \mathrm{mg} / \mathrm{dl}$ ) e alterações neurológicas, auditivas, visuais, de linguagem e de desenvolvimento ${ }^{3-4}$.

Entretanto, em 2001, Sugama et al. ${ }^{5}$ questionaram a rara ocorrência de encefalopatia bilirrubínica em prematuros, ao detectarem kernicterus em dois pacientes de 31 e 34 semanas de idade gestacional, respectivamente com bilirrubinemia de 13,1 e 14,7 mg/dl, sendo que nenhum deles apresentava sintomatologia sugestiva da doença no período neonatal. O primeiro caso tinha quadro de paralisia cerebral atetóide aos 3 anos de idade, e o segundo não sustentava a cabeça aos 7 meses e tinha hipotonia de tronco. A ressonância magnética evidenciou alterações compatíveis com encefalopatia bilirrubínica, levando à 